\title{
Musculação e o uso de esteroides anabolizantes
}

\author{
Bodybuildling and the use of anabolic steroids \\ Culturismo y el uso de esteroides anabólicos
}

Recebido: 06/10/2021 | Revisado: 14/10/2021 | Aceito: 19/10/2021 | Publicado: 22/10/2021

\author{
Ana Caroline Corrêa Costa \\ ORCID: https://orcid.org/0000-0002-5577-7381 \\ Faculdade Integrada Carajás, Brasil \\ E-mail: ana_caroline.98@hotmail.com \\ Eduarda Moreira Lima \\ ORCID: https://orcid.org/0000-0002-5933-8888 \\ Faculdade Integrada Carajás, Brasil \\ E-mail: eduardamoreira0911@ gmail.com \\ Jânio Sousa Santos \\ ORCID: https://orcid.org/0000-0003-2180-1109 \\ Faculdade Integrada Carajás, Brasil \\ E-mail: santosjs.food@gmail.com
}

\begin{abstract}
Resumo
Esse trabalho teve por finalidade propor uma discussão acerca do uso de esteroides anabolizantes, sem acompanhamento de um profissional qualificado, no cenário da musculação. Trata-se de uma pesquisa bibliográfica que usa como ponto de partida a idealização do corpo perfeito, propagado pelas mídias sociais e buscado por praticantes de musculação, que utilizam os anabolizantes como forma mais rápida de obter o objetivo desejado. Como resultado da pesquisa, os conceitos, composições das substâncias, bem como a descrição e finalidade das mais utilizadas, permitem ver o quão agressivos os efeitos colaterais podem ser ao corpo humano, quando utilizadas de forma indiscriminada. Conclui-se nesse sentido, que é perceptível a importância de trabalhos com essa temática para profissionais, adeptos da musculação e até mesmo para a população como todo, de forma que seja compreendido a necessidade de orientação por profissionais capacitados para indicação de uso das substâncias, tendo em vista os diversos efeitos colaterais desencadeados pelo seu uso.
\end{abstract}

Palavras-chave: Esteroides; Anabolizantes; Musculação; Efeitos Colaterais.

\begin{abstract}
This work aimed to propose a discussion about the use of anabolic steroids, without monitoring by a qualified professional, in the bodybuilding scenario. This is bibliographical research that uses as a starting point the idealization of the perfect body, propagated by social media and sought by bodybuilders, who use anabolic steroids as a faster way to achieve the desired goal. As a result of the research, the concepts, compositions of substances, as well as the description and purpose of the most used ones, allow us to see how aggressive the side effects can be to the human body, when used indiscriminately. In this sense, it is possible to conclude that the importance of work with this theme for professionals, fans of bodybuilding and even for the population as a whole is noticeable, so that the need for guidance by trained professionals to indicate the use of substances is understood, in view of the various side effects triggered by its use.
\end{abstract}

Keywords: Steroids; Anabolics; Bodybuilding; Side Effects.

\section{Resumen}

Este trabajo tuvo como objetivo proponer una discusión sobre el uso de esteroides anabólicos, sin seguimiento por un profesional calificado, en el escenario del culturismo. Se trata de una investigación bibliográfica que utiliza como punto de partida la idealización del cuerpo perfecto, propagada por las redes sociales y buscada por los culturistas, que utilizan los esteroides anabólicos como una forma más rápida de lograr el objetivo deseado. Como resultado de la investigación, los conceptos, composiciones de sustancias, así como la descripción y finalidad de las más utilizadas, permiten ver cuán agresivos pueden ser los efectos secundarios para el cuerpo humano, cuando se usan de forma indiscriminada. En este sentido, es posible concluir que se nota la importancia que tiene el trabajo con esta temática para los profesionales, aficionados al culturismo e incluso para la población en su conjunto, por lo que es evidente la necesidad de orientación por parte de profesionales capacitados para indicar el uso de sustancias. Entendido, en vista de los diversos efectos secundarios provocados por su uso.

Palabras clave: Esteroides; Anabólicos; Culturismo; Efectos secundarios. 


\section{Introdução}

Nos últimos anos as mídias sociais tem contribuído para a idealização de um corpo "perfeito", criando uma importância exagerada acerca da aparência física. Nesse sentido a atividade física vem atraindo cada vez mais jovens a frequentarem centros desportivos e academias com o propósito de buscarem o corpo ideal (Bezerra \& Silva, 2019).

A musculação é um tipo de atividade física que envolve cargas e tem conquistado bastante adeptos. Seus resultados de desenvolvimento corporal por parte dos praticantes tendem a ser gradativos, ocasionando muitas vezes, uma não percepção do processo de ganhos de massa muscular. Nesse contexto, a busca por melhor desempenho para o alcance de bons resultados, tem feito com que indivíduos se utilizem de recursos extremos, como uso de esteroides anabolizantes, sem prescrição e acompanhamento de um profissional. Tais ações ocorrem pela busca incansável de um corpo esteticamente atraente, que apresente volume muscular com o mínimo de gordura possível (Venâncio, Nobrega, Tufik \& Mello, 2010).

A palavra esteroide significa "óleo sólido" e se origina do grego "stereos", que significa sólido e do latim "oleum" que significa óleo (Wilmore \& Costill, 2001). Os esteroides anabolizantes são substâncias que viabilizam o aumento do volume muscular a curto prazo. Segundo Mcardle (2003), são substâncias em geral utilizadas em combinação com treino, tendo em vista sua capacidade de aprimoramento de força, velocidade e potência nos treinos.

Inicialmente, essas substâncias foram desenvolvidas para serem utilizadas no tratamento de diversas patologias. No entanto seu uso tem sido elevado entre jovens praticantes de musculação com fins estéticos e sem o devido acompanhamento profissional. Além disso, há estudo demonstrando que usuários não sabem dos reais riscos à saúde que o uso inadequado dos mesmos pode causar (Oviedo, 2013).

Vale ressaltar que, o uso indiscriminado e sem recomendação e/ou orientação profissional pode gerar transtornos a saúde. Assim, faz se necessário um olhar mais criterioso acerca do tema, no sentido de fornecer informações relevantes e científicas sobre o uso dos esteroides anabolizantes (Oviedo, 2013). Rocha (2007) ressalta que o uso apenas para estética pode causar vários danos e prejuízos a saúde. Esses danos podem ser apresentados tanto em órgãos como em tecidos, vindo a causar eventuais problemas ao sistema cardíaco, estimular a agregação de plaquetária e alteração dos níveis de colesterol circulante. Ou seja, o uso dessas substâncias deve ser realizado sob orientação de um profissional capacitado para compreender as reais necessidades na administração de anabolizante no organismo. Porém, essa realidade não está próxima do que se vê atualmente.

Tendo em vista esse cenário, o uso indevido dos esteroides anabolizantes, tornou-se um problema de saúde pública, pois, há uma preocupação quanto ao fácil acesso por parte da população. Dessa maneira, entende-se que estudos voltados para a orientação e conscientização das substâncias anabolizantes necessitam ser mais desenvolvidos e, principalmente, divulgados à população, para que a mesma tenha total esclarecimento dos possíveis efeitos colaterais causados pelo uso destes recursos.

\section{Metodologia}

O presente trabalho traz como procedimento metodológico a revisão narrativa, descrita como pesquisa bibliográfica. O mesmo segue orientação de Estrela (2018), que fornece suporte metodológico, permitindo uma melhor compreensão sobre os aspectos a serem considerados na elaboração do trabalho.

A pesquisa foi sequencialmente realizada, com a finalidade de alcançar os objetivos propostos. Nesse sentido, primeiramente será determinado o objeto de estudo e os objetivos da pesquisa, seguido do levantamento bibliográfico, que tem como intuito de ratificar os objetivos da pesquisa por meio da investigação de estudos sobre o uso de esteroides anabolizantes em praticantes de musculação, bem como a abordagem do tema, sob a análise de estudiosos.

A coleta de dados em documentos científicos foi realizada por meio de buscas nas bases de dados eletrônica como: Biblioteca Virtual em Saúde (BVS BRASIL), Scientific Eletronic Library Online (Scielo) e o portal de periódico da Coordenação de Aperfeiçoamento de Pessoal de Nível Superior (CAPES). Foi definido o recorte temporal a partir do ano de 
2000 até o ano atual (2021). Utilizou-se como descritores para busca dos documentos as seguintes palavras-chave: Esteroides anabólicos, Anabolizantes, Treinamento de força, Musculação.

Em seguida, foram selecionados os artigos mais relevantes, e realizada as extrações de dados sobre a temática, permitindo melhor análise e apontamentos para as abordagens em torno do uso de anabólicos no cenário da musculação. Bem como a importância das informações sobre uso e efeitos colaterais dessas substâncias.

\section{Resultados e Discussão}

\section{Musculação}

A musculação tem se tornado cada vez mais popular e seus praticantes tem aumentado significativamente nos últimos anos (Chagas \& Lima, 2004). A busca da prática da musculação se dá majoritariamente pelo ganho de massa muscular, melhoria postural, redução do percentual de gordura corporal ou até mesmo para finalidade estética. Mas, há uma padronização de corpo ideal imposta pela sociedade. A qual é fortalecida pelas mídias sociais, que leva as pessoas a procurarem manter o corpo considerado perfeito. Devido a este fato, a procura pelas academias tem aumentado e o acesso às mesmas tem se tornado mais fácil, pois elas estão presentes em vários locais e atendem às diversas faixas etárias (Reis, 2004). O que pode ser confirmado pela afirmação de Lima (2009) que a procura pela prática da musculação, entre outros motivos tem relação com o aumento dos locais para praticá-la, ou seja, atualmente é mais acessível locais específicos para prática de musculação.

A musculação é realizada majoritariamente em academias, tendo como predominância o treino de força muscular, enfatizando a hipertrofia. Corsino (2007) relata que, é importante ressaltar que o treinamento de força por meio da musculação, proporciona ao indivíduo um aumento da força, hipertrofia da massa corporal magra, aumento da massa óssea e a melhoria das funções física, mental e psicológica. Estes benefícios possibilitam a redução do risco de doenças ortopédicas, osteoporose, doenças coronarianas, diabetes e a obesidade.

A força muscular é uma capacidade física cuja manifestação depende de fatores tanto estruturais quanto neurais, hormonais ou até mesmo psicológicos (Badillo \& Ayestarn, 2004). Schoeneld (2010) diz que o ganho de força depende da otimização de adaptações durante o treinamento. Essas adaptações seguem um padrão temporal específico, ou seja, quando ocorre um estímulo agudo ao exercício, ocorre uma adaptação no corpo. Porém, somente ocorrerá uma mudança celular, tecidual ou em um sistema específico, se houver uma repetida exposição ao estímulo naquele programa de treinamento. Segundo os mesmos autores, a adaptação ao treinamento resulta em última análise se um programa de treinamento de força é eficaz e se um atleta é capaz de atingir um nível mais alto de função fisiológica ou de desempenho.

$\mathrm{Na}$ prática da musculação ocorre o aumento da força muscular, que apresenta como principal modificação morfológica a hipertrofia muscular, definida como um aumento do volume de um determinado músculo em consequência do aumento da área de secção transversa das fibras que o constituem (Crozeta \& Oliveira, 2009). As fibras musculares aumentam de tamanho dependendo da quantidade e qualidade das proteínas actina e miosina, responsáveis pela contração muscular. Para que se possa averiguar um aumento significativo da fibra muscular, torna-se necessário um período mais longo de treinamento, o que possibilita um aumento na secção transversa do músculo.

Assim, com o intuito do aumento da massa muscular em um curto prazo de tempo, atletas começaram a fazer uso dos Esteroides Anabolizantes Androgênicos (EAA) sendo incialmente utilizados entre levantadores de peso e fisiculturistas, expandindo-se assim para outros esportes, como a musculação (Lima \& Cardoso, 2011).

\section{Esteroides Anabolizantes}

Para entender melhor a presente pesquisa é preciso se aprofundar um pouco sobre a visão de alguns teóricos que discutem sobre o lúdico como uma ferramenta eficaz dentro do processo de ensino, levando em consideração um bom 
planejamento de acordo com o nível de ensino, que na presente pesquisa, destaca-se a Educação Infantil.

Os esteroides são hormônios que possuem uma estrutura química similar à do colesterol e a maioria deles deriva do colesterol. Devido a este fato, são classificadas como substâncias lipossolúveis e que se difundem facilmente através das membranas celulares. Sendo, uma classe de lipídios que possuem uma estrutura em comum de hidrocarbonetos com 17 átomos de carbonos ligados em quatro estruturas cíclicas (Wilmore \& Costill, 2001).

Partindo do núcleo básico, é possível observar a formação de outras estruturas químicas. Isso se dá, porque a diferença de um esteroide para o outro está nos grupos funcionais que se ligam a esse núcleo central, que podem ser álcoois, ésteres, enóis, cetonas, ácidos carboxílicos e outros. Entre os esteroides naturais mais importantes encontra-se o colesterol e seus derivados, como, o estradiol (principal hormônio sexual feminino) e a testosterona (principal hormônio sexual masculino), que podem ser vistos respectivamente na Figura 1.

Figura 1 Estrutura química do Colesterol (A), Estradiol (B) e Testosterona (C).

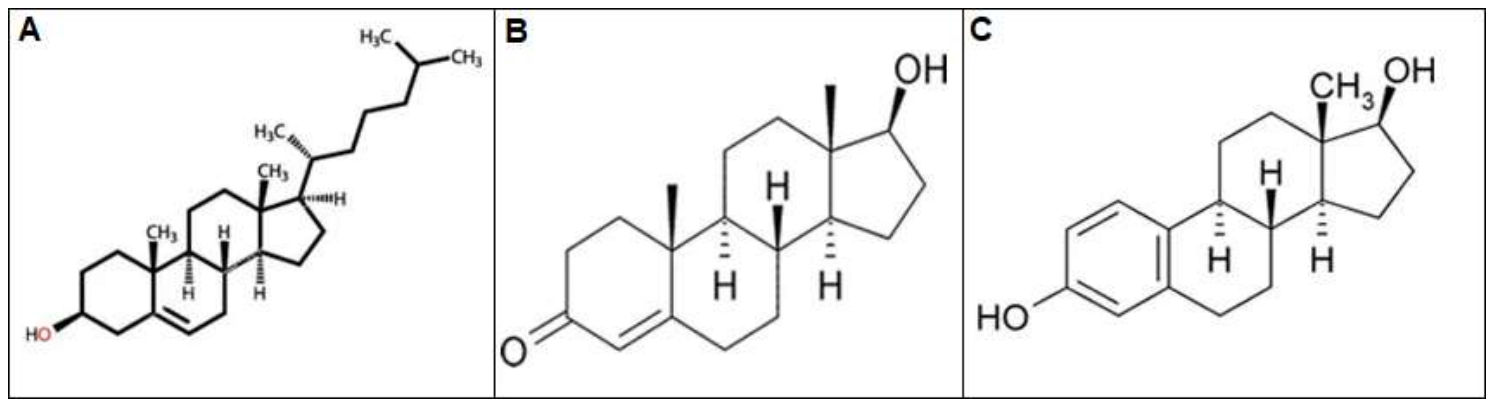

Fonte: Soares (2013).

Quimicamente os hormônios são divididos em três classes: peptídicos ou proteínas, derivados lipídicos e derivados de aminoácido (Sousa, 2002). Os derivados lipídicos se dividem em dois grupos: os hormônios esteroides e os eicosanoides. Os hormônios esteroides são derivados do colesterol. Os eicosanoides são derivados de um ácido graxo. Os derivados de aminoácidos são os mais simples. Os peptídicos ou proteínas consistem de cadeias de aminoácidos, entre 3 a 200 (Tortora, 2017).

No início dos anos 50, os esteroides anabólicos obtiveram grande significância em relação a sua utilização pela medicina, que se voltava para tratamento de pacientes deficientes na produção de estrogênios ou que sofriam de doenças caracterizadas pelo desgaste muscular. Além disso, os esteroides anabolizantes foram administrados às crianças com padrões de crescimento retardado, já que aceleram o crescimento pelo aumento da velocidade de maturação óssea e pelo desenvolvimento de massa muscular (Wilmore \& Costill, 2001).

Atualmente beneficia no tratamento de patologias relacionadas com o hipogonadismo, deficiência do metabolismo proteico, deficiência de testosterona, alguns tipos de anemia e de câncer de mama, controle da menopausa e andropausa (Machado, apud Alves Sobrinho, Rossi Junior, Corsini, Soares, \& Esteves, 2020).

De acordo com Lima (2009), os anabolizantes são prescritos como remédios, com o intuito de prevenir doenças, ou reestabelecer a saúde fisiológica. Então, são dimensionadas doses consideradas terapêuticas, para possibilitar que o organismo funcione normalmente. Dessa maneira, a forma mais segura de uso é por meio de receitas médicas, indicando tratamento para distúrbios de baixa produção hormonal pelo corpo (Barquilha, 2009).

\section{Classificação dos Esteroides}

A Base Nacional Comum Curricular veio para agregar qualidade a mediação do ensino, este documento importante em seu conteúdo, traz habilidades e objetivos que possibilitam ao profissional docente ter um ponto de partida bem 
fundamentado para realizar o planejamento e uma boa execução de suas aulas.

Ao analisar o termo "esteroides anabolizantes", tem-se que esteroide se refere à classe atribuída dessas drogas, enquanto anabolizante refere-se às substâncias existentes que promovem o anabolismo (Roque, et al., 2007).

Existem basicamente três categorias de esteroides: estrógenos, andrógenos e cortisona. Os estrógenos (hormônios femininos) são produzidos principalmente no ovário em mulheres, em pequenas quantidades no testículo dos homens e nas glândulas adrenais em ambos os sexos. Já os andrógenos (hormônios masculinos) são sintetizados principalmente nos testículos, em quantidade inferiores no ovário e nas glândulas suprarrenais. Enquanto que a cortisona, esteroide produzido por ambos os sexos com efeito anti-inflamatório e analgésico (Guimarães, 2009).

\section{Derivados da Testosterona}

Segundo Heartgens e Kuipers (2004), os Esteroides Anabólicos Androgênicos basicamente são derivados sintéticos do hormônio testosterona. Os andrógenos representam a classe em que estes hormônios estão inseridos, e são produzidos principalmente pelo testículo, cujo principal hormônio sintetizado é a testosterona.

Os autores completam ao afirmar, que a testosterona em si não é eficaz quando tomada via oral ou injeção, por conta do metabolismo realizado pelo fígado, onde parte da substância deixa de ser aproveitada pelo corpo, por isso foi modificada para ser melhor aproveitada. Nesse sentido, alguns compostos orgânicos podem ser ligados à testosterona, de forma a modificá-la, como é o caso dos ésteres, responsáveis por modificar o tempo de meia vida dos anabolizantes, ou seja, o tempo de ação do anabolizante dentro do corpo. Estes podem ser curtos, com tempo de ação do anabolizante menor, ou longo, com tempo de ação maior. Entre os mais conhecidos, estão: decanoato (29 carbonos), enantato (26 carbonos), cipionato (27 carbonos) e proprionato (22 carbonos), como indicados na Figura 2.

Figura 2 Compostos orgânicos ligados à testosterona.

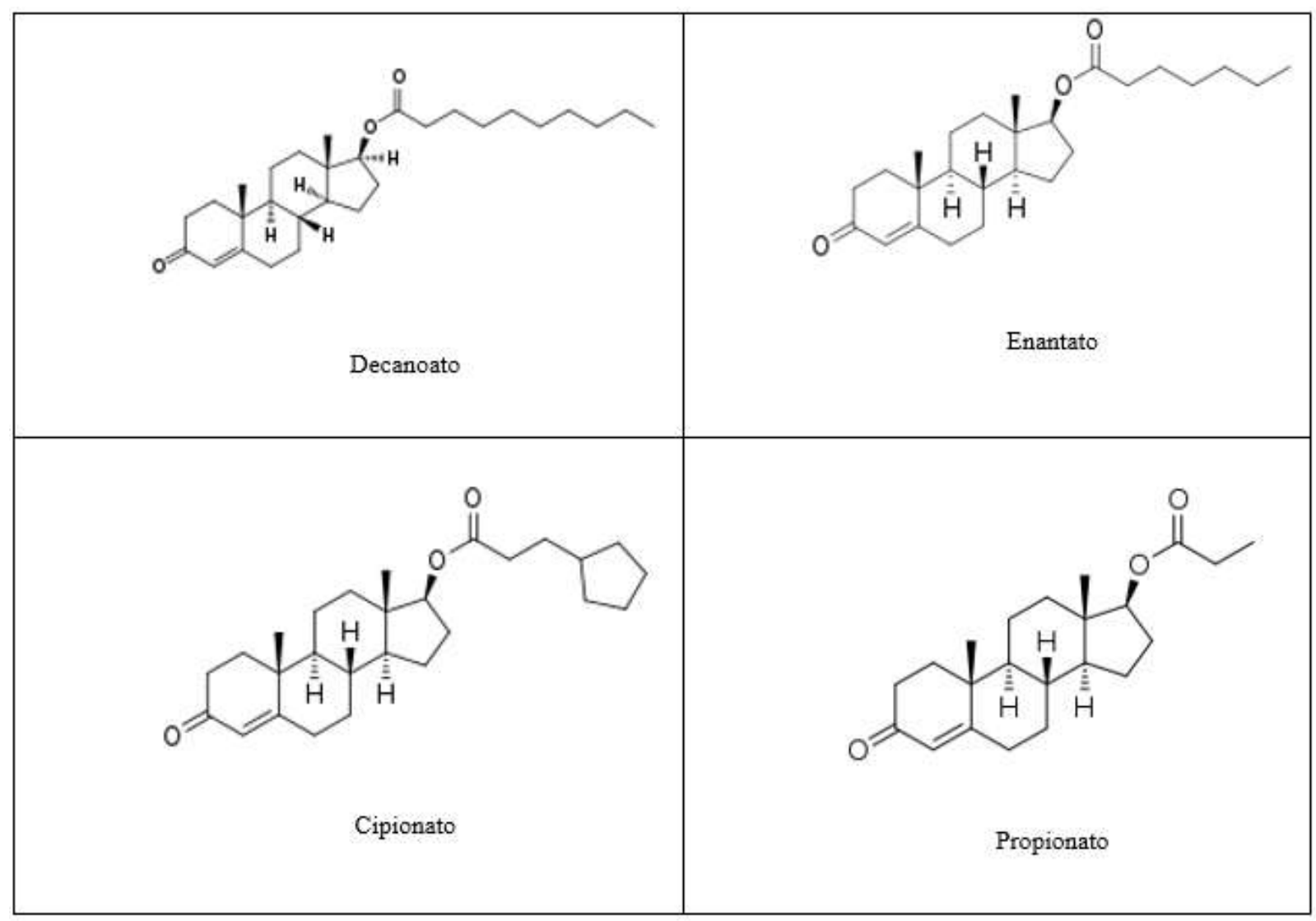

Fonte: Soares (2013). 
Vale dizer que, o proprionato preserva a meia-vida da testosterona em bem menos tempo (de 1 a 3 dias), do que um éster de enantato (4 a 5 dias), decanoato (6 a 7 dias) ou cipionato (8 dias), por exemplo (Garcia, 2014).

Os esteroides anabolizantes androgênicos, portanto, possuem a característica de promover o anabolismo, além das características masculinizantes. A testosterona, em sua forma natural e produzida pelo organismo. É um hormônio benéfico devido sua influência no metabolismo do corpo em aumentar a massa magra, força muscular, acelerar a recuperação tecidual e regular o acúmulo de gordura (Abrahin \& Sousa, 2013).

\section{Derivados da progesterona}

Os principais hormônios sexuais femininos são o estrógeno e a progesterona, produzidos principalmente pelos ovários durante a vida reprodutiva. O estrógeno, também conhecido como estrogênio, é um hormônio sexual feminino produzido pelos folículos do ovário desde a adolescência até a menopausa. Além dos ovários, o estrógeno pode ser reproduzido pelo tecido adiposo, células da mama e ósseas e glândula adrenal (Abrahin \& Sousa, 2013).

Enquanto que a progesterona, é um hormônio, produzido no corpo lúteo, nos ovários, e é responsável pela diferenciação do endométrio, controle da implantação e maturação do epitélio mamário, sendo, portanto, essencial para o início e a manutenção

da gestação (Oliveira, 2016). Abrahin e Sousa (2013) reforçam ainda o papel da progesterona no processo da gravidez, afirmando que a mesma é responsável por regular o ciclo menstrual e preparar o útero para receber o óvulo fertilizado, evitando que este seja expulso pelo corpo.

Como ação direta no organismo, os estrógenos estimulam o crescimento das células musculares e está diretamente relacionado com o desenvolvimento das características sexuais femininas. Mas, se apresenta nos homens, produzido, em pequenas quantidades, pelos testículos, células ósseas, tecido adiposo e glândula suprarrenal (Guimarães, 2009).

Nas mulheres, o estrógeno é produzido, principalmente, pelos ovários, e está relacionado ao ciclo menstrual. Sua síntese começa através do estímulo de dois hormônios produzidos pela hipófise no cérebro, o LH (hormônio luteinizante) e o FSH (hormônio folículo estimulante), que enviam sinais aos ovários para produzir o estradiol, que é o tipo de estrógeno mais potente produzido durante toda a idade reprodutiva da mulher. Esses hormônios participam do ciclo menstrual, no qual haverá a formação do ovócito e a produção dos hormônios ovarianos estrógeno e progesterona, que preparam o útero para uma possível gravidez (Oliveira, 2016).

Há ainda outros dois tipos de estrógeno que podem ser produzidos, como a estrona e o estriol. Esses, são menos potentes e não necessitam do estímulo dos hormônios cerebrais, pois durante a gravidez, as células adiposas, da mama, óssea e dos vasos sanguíneos, glândula suprarrenal e a placenta, produzem uma enzima capaz de transformar o colesterol em estrógeno (Oliveira, 2016).

\section{Formas de Administração}

Frente à tecnologia apresentada hoje, uma substância anabolizante pode ser administrada de diversas maneiras, como comprimidos, gel, cápsulas, além de injetáveis, sendo estes solúveis em água ou em meio oleoso (Neto, 2002).

Lima e Cardoso (2011) diz que a utilização das substâncias geralmente é oral ou parenteral, porém já foram relatados casos de ingestão das mais variadas formas como via retal, nasal e transdérmica ou implante de cápsulas para suplantar o metabolismo. Mas, é importante ressaltar que a forma oral e injetável são as mais usuais.

\section{Via oral}

As substâncias orais, se apresentam como cápsulas, comprimidos ou em forma líquida, e são ingeridas com auxílio de 
líquidos ou adicionado na alimentação. Vale ressaltar que tratando-se das substâncias ingeridas por via oral, a prejudicação ao aparelho gastrointestinal, variam de grau do $\mathrm{pH}$ da substância, bem como suas correlações e outros (Carvalho, Fortunato \& Rosenthal, 2007).

Nesse sentido, é importante frisar que as substâncias, para serem ingeridos por via oral, não podem apresentar deterioração no sistema gastrointestinal e/ou fígado. Isso porque ao ser ingerida a droga passa pelo estomago, é absorvida no intestino e processada pelo fígado, de onde é levada para a corrente sanguínea. Esse processo sobrecarrega o fígado que acaba sendo danificado pelo esforço para combater algo que não consegue processar completamente (Souza, 2002).

Devido a isso, as substâncias sofrem degradação ao passar pelo sistema gastrointestinal, fator pelo qual criaram as substâncias 17-alfa-alquilado,que consiste na adição de um grupo alquilo de uma molécula a outra no carbono 17 e posição alfa, permitindo que a substância resista, sem degradação, ao passar pela metabolização hepática (Santos, 2010).

Assim, a quantidade de ingestão, não é a mesma do que será absorvida pelo organismo, tendo em vista que há alterações na absorção, a depender da qualidade da substância, das características fisiometabólicas de cada indivíduo, bem como do comportamento do organismo como resposta ao uso, dentre outros fatores importantes no processo (Carvalho, Fortunato \& Rosenthal, 2007).

\section{Injetável}

As substâncias ergogênicas hormonais injetáveis apresentam-se em caráter diluído (Sal da substância + Sal do éster + Soluto) em meio aquoso ou oleoso. Os dissolvidos em água, também conhecidos como suspensão aquosa, são assim denominados por necessitarem ser agitados para tornar a mistura água/esteroide homogênea, não tendo grande utilização entre usuários por serem mais suscetíveis a bactérias, ao passo que os da base oleosa possuem agentes antibacterianos mais eficientes (Mota, 2010). O mesmo autor ainda afirma que, há substâncias que podem também ser tanto diluída em água quanto em óleo, ao mesmo tempo, como é o caso da testosterona suspensão (diluída em água) e o Cipionato de testosterona (diluído em óleo).

Os anabolizantes injetáveis apresentam uso normalmente intramuscular, passando direto para a corrente sanguínea. Apesar dessa forma de administração da substância aparentar ser menos ofensivos ao corpo humano, estas provocam mais toxidade para os rins (Souza, 2002).

Vale dizer que, quando se faz uma aplicação com essas substâncias ela é absorvida e metabolizada em diferente tempo, variando de acordo com a sua especificidade e de acordo com o éster utilizado (Mota, 2010).

\section{Esteroides anabolizantes mais usados}

Os anabolizantes em sua maioria são derivados dos hormônios testosterona e progesterona, podendo ser administrado de forma oral ou injetável. Nesse sentido, percebe-se que há uma gama dessubstancias disponíveis no mercado.

Tendo em vista esse cenário, segue a vista dos anabolizantes mais comumente utilizados para melhoria de performance e estética entre os praticantes de musculação.

\section{Estanozolol}

Entre os anabolizantes mais utilizado em larga escala na atualidade por jovens e adultos, de ambos os gêneros com a finalidade de ganho de massa muscular e melhora no condicionamento físico está o estanozolol (EST) (Silva, et al, 2007). Isso porque, esse esteroide anabolizante age na reconstrução dos tecidos, aumentando o apetite e estimulando o ganho de massa corporal.

O EST é um esteroide anabolizante sintético derivado da testosterona, e uma de suas características é que pode ser 
administrado tanto injetável, por via intramuscular, quanto por via oral.

Clinicamente, sua utilização é voltada para o tratamento de anemia, angioedema hereditário, estados de depreciação física, fraturas de lenta consolidação, osteoporose, queimaduras extensas, raquitismo, anorexia e no tratamento de doenças crônicas e debilitantes, osteoporose, queimaduras extensas, fraturas de recuperação lenta, retardo de crescimento em crianças, deficiências andrógenas, algumas anemias raras, síndromes asmáticas ou artrite reumatoide (Grunding \& Bachman, 1995 apud Santos, 2007).

Entre os efeitos colaterais do estanozolol está a náusea, vômito, acne, insônia ou excitação, podendo levar ainda a sérias complicações renais, hepáticas, neurológicas, entre outras, devido a sua elevada toxicidade (Silva, et al, 2007).

\section{Durateston}

O Durateston ou simplesmente "dura", é um dos anabolizantes a base de testosterona mais consumido no mundo. É um esteroide injetável que combina a ação de quatro ésteres de testosterona (proprionato, fenilpropionato, isocaproato e decanoato), que se misturam para obter ação imediata após a aplicação e mantê-la por longo período (Gruding, apud Santos, 2007).

Essa junção ocorre, tendo em vista que o proprionato tem ação imediata por curto período; o fenilpropionato e o isocaproato têm princípio de ação mais lento, e maior duração e o decanoato tem ação mais potente e prolongada.

Este medicamento possibilita o crescimento muscular, além da diminuição da gordura corporal do indivíduo. Sua ação é muito mais intensa e, por ser lipossolúvel, a testosterona entra no organismo e no citoplasma da célula, ligando-se a um receptor. $\mathrm{O}$ uso resulta no aumento de força e ganho de peso e não provoca retenção hídrica como a maioria dos esteroides altamente androgênicos. (Gruding \& Bachman 1995 apud Santos, 2007).

E é indicado no tratamento de reposição de testosterona em homens portadores de condições associadas com hipogonadismo após castração química, eunucoidismo, hipopituitarismo, impotência endócrina, entre outras situações.

Entre os efeitos colaterais ocorridos durante o tratamento com a substância são priapismo e outros sinais de estimulação sexual excessiva, oligospermia e diminuição do volume ejaculatório e retenção de líquidos (Abril et al., 2005, Bonetti et al., 2008).

\section{Decanoato de nandrolona}

O decanoato de nandrolona, é um esteroide injetável, diluído em óleo de amendoim. Essa droga se configura como um dos EAA mais procurados no mundo (Kutscher, Lund \& Perry, 2002, apud Lima, 2018).

A nandrolona atua no aumento da massa muscular e é preferida pelos atletas que abusam das doses, porque é pouco androgênica, se comparada à testosterona, isto é, não atua muito na esfera sexual, o que reduz os efeitos colaterais mais indesejáveis pelos consumidores, além de apresentar alto potencial anabólico (Lima, 2018).

A substância ainda é indicada no tratamento de doenças debilitantes crônicas, ou trauma. Mas, o uso prolongado de esteroides resulta em quadros clínicos mais severos, tais como, azoospermia, hipertrofia prostática e carcinoma prostático (Pedroso 2014).

Os principais efeitos adversos do decanoato de nandrolona são a virilização em mulheres, hipertrofia clitoriana e amenorreia, enquanto os homens apresentam acne e a inibição da espermatogênese (Ribeiro apud Braga, 2005).

\section{Oxandrolona}

A oxandrolona, é bastante utilizado por ser moderadamente andrógena e apresentar bom resultado anabólico, sem efeitos colaterais pronunciados. E tem como efeito principal o grande aumento de força, por ampliar os depósitos de fósforo 
creatina intracelular (Gallaway, 1997 apud Santos, 2007).

Como fármaco, é utilizada para ajudar pacientes que perderam a força muscular, no tratamento de hepatite alcoólica, má nutrição calórica proteica moderada, falha no crescimento físico e em pessoas com síndrome de Turner (Santos, 2007).

E os efeitos colaterais mais comuns durante o tratamento com oxandrolona está o aparecimento de características sexuais secundárias masculinas em mulheres, irritação da bexiga, sensibilidade ou dor nas mamas, desenvolvimento das mamas nos homens, priapismo e acne.

Além disso, embora seja mais raro, pode ainda ocorrer disfunção hepática, diminuição dos fatores de coagulação, aumento do cálcio no sangue, leucemia, hipertrofia da próstata, diarreia e alterações do desejo sexual (Santos, 2007).

\section{O cipionato de testosterona}

O cipionato de testosterona, é um éster injetável de base oleosa, para uso intramuscular, conhecido por promover rápido ganho de força e volume muscular. É altamente androgênica e apresenta boas propriedades anabólicas.

O medicamento é usado como um anabolizante, por pessoas que buscam a promoção muscular, por provocar crescimento muscular de forma mais rápida que os métodos convencionais de musculação e dieta. Isso porque a droga promove o ganho de massa magra, aumenta a resistência física e força e melhora a densidade óssea.

Mas, além dos benefícios estéticos, o medicamento atua no tratamento de distúrbio na produção de testosterona, como doença nos testículos ou hipogonadismo primário, favorece a produção de espermatozoides, trata a falta de apetite sexual, cansaço permanente e outros problemas.

Quando administrado em dosagens inadequadas e de forma prolongada, pode provocar alguns efeitos colaterais, como: Atrofia dos testículos; Insônia; Ginecomastia; Queda de cabelos; Náuseas intensas; Perda da libido; Problemas renais; Retenção de líquido; Câncer de fígado; Dor no local da aplicação (Gruding \& Bachman, 1995 apud Santos, 2007).

\section{Oximetalona}

A oximetalona é um esteroide anabolizante de uso via oral, apresentado e forma de comprimidos metabolizado pelo fígado, trazendo seus efeitos positivos rapidamente.A substância é muito utilizada por aqueles que tem dificuldade em formar massa muscular logo no início de seu treinamento. Por apresentar essas características, é conhecido como um dos esteroides mais potentes que existem e bastante conhecido por sua habilidade em gerar rápido aumento de peso e força no usuário.

Em dose terapêuticas, sua utilização era voltada para tratar diferentes tipos de anemias em estágios muito graves, e indicado em terapias para o tratamento de anemia causada pela produção deficiente de eritrócitos. Além disso, é indicado para osteoporose e problemas crônicos que causam perda de tecido muscular (como HIV/AIDS).

Vale dizer que o hemogenin é muito tóxico ao fígado e causa retenção de água, acne e perda de cabelo. Além destes sintomas, o uso da droga acarreta a ginecomastia, dores de cabeça, de estômago e outros problemas (Gallaway, 1997 apud Santos, 2007).

\section{Efeitos adversos dos esteroides anabolizantes}

Como já dito, o uso dos anabolizantes tem fins terapêuticos, mas, é importante frisar a necessidade de um acompanhamento profissional. Segundo Lima (2009), o uso de doses acima das terapêuticas, pode gerar riscos à saúde, por meio de reações adversas. Assim, Ribeiro (2008) afirma que quanto maior a quantidade e a dose de EAA's, aumenta a possibilidade de aparecimento de efeitos colaterais em diferentes órgãos do sistema. Estudos apontam que entre os principais efeitos colaterais pelo uso excessivo da substância estão a alteração no processo natural de formação muscular (Kersey, et al.,

2012). Especula-se ainda que os esteroides anabolizantes afetam o equilíbrio hormonal de duas formas. Primeiro, o 
anabolizante restabelece os níveis de testosterona após o exercício acelerando a regeneração muscular, e em seguida, bloqueia os efeitos regenerativos dos glicocorticoides (Oviedo, 2013).

Vale ressaltar que o desenvolvimento dessas enfermidades varia de indivíduo para indivíduo, tipo de esteroide consumido, do tempo de uso, quantidade de esteroide utilizado e da predisposição de cada um em desenvolver tais efeitos colaterais (Grinsopoon, 2006). Para Michalik (2006), a curto prazo, os esteroides desenvolvem o corpo, mas a longo prazo o preço pode ser alto. Externamente o usuário de esteroide pode parecer mais desenvolvido corporalmente, mas, internamente as consequências podem ser fatais.

\section{Efeitos no sistema cardiovascular}

No sistema cardiovascular, o uso de esteroides aumenta os riscos de doenças cardíacas, pela elevação dos níveis de colesterol (Santos, 2007). Entre as alterações induzidas pelos esteroides anabolizantes que podem predispor ao desenvolvimento de doenças cardiovasculares pode-se citar o hiperinsulinismo e uma tolerância alterada à glicose, redução dos níveis de colesterol HDL e aumento da pressão arterial (Santos, 2007).

A diminuição significativa no HDL é um problema bastante comum em usuários crônicos dos anabolizantes. As baixas concentrações dessa lipoproteína estão associadas a um risco mais alto da doença arterial coronariana e de infarto do miocárdio. Outra doença desencadeada nos usuários crônicos, é a cardiomiopatia, doença que compromete os músculos cardíacos, podendo levar ao infarto (Wilmore \& Costil, 2006). Outro problema comum é a hipertensão, que provoca dores de cabeça, insônia e dificuldades respiratórias. Além desses efeitos negativos, os EAA parecem inibir alguns dos efeitos positivos do exercício sobre o sistema cardiovascular, como a diminuição de frequência cardíaca em repouso (Carmo et al., 2011).

De um modo geral, os anabolizantes podem comprometer a saúde e a função do sistema cardiovascular, aumentando o risco de acidente vascular cerebral e de infarto do miocárdio. (Mcardle, Katch \& Katch, 2008).

\section{Efeitos no sistema digestivo}

No sistema digestivo, o órgão mais afetado pelo uso dos anabolizantes é o fígado, que faz a metabolização dos carboidratos, lipídeos e proteínas, além da secreção de bile e sais biliares (Tortora \& Grabowski, 2006). Nesse sentido, as moléculas de testosterona dos anabolizantes não são facilmente metabolizadas pelo fígado, tendo que passar várias vezes pelo fígado antes de serem modificadas, acarretando assim uma série de danos a este órgão.

Entre problemas mais frequentes desencadeados pelo uso dos anabolizantes, do ponto de vista hepático, estão: estão descritos vários efeitos nomeadamente: colestase intra-hepática, hepatite, adenoma e carcinoma hepatocelular (Brinquinho; Sousa, Resende \& Valente, 2017).

Os anabolizantes levam a uma inflamação no fígado e, consequentemente, induzem o desenvolvimento de quadro conhecido como colestase, dificuldade para o escoamento da secreção de bile, e amarelidão, causada pela icterícia, podendo haver remissão da icterícia após a interrupção do uso da droga (Brinquinho, Sousa, Resende \& Valente, 2017).

Além disso, o uso das substâncias pode levar ainda ao câncer primário do fígado, quando acomete um fígado normal, sem desenvolvimento inicial da cirrose. Isso porque, entre as origens do câncer primário do fígado não cirrótico está o uso de anabolizantes e o desenvolvimento de infecção pelo vírus da hepatite B, podendo essas duas condições levar ao aparecimento de um tumor no fígado. Estes tumores são geralmente benignos, mas têm sido observadas associação de tumores malignos desencadeado pelo uso dessas drogas. (Socas et al., 2005).

\section{Efeitos no sistema reprodutor}

Os esteroides anabolizantes envolvem a testosterona e seus derivados, são produzidos no córtex adrenal e gônadas 
(ovários e testículos) e geram as características sexuais acessórias agregadas à masculinidade (Silva, Danielski \& Czepielewski, 2002).

$\mathrm{Na}$ mulher, o estrógeno é produzido nos ovários, enquanto nos homens o andrógeno é produzido pelo testículo, caracterizando respectivamente as identidades feminina e masculina, com predominâncias diferentes (Fonseca et al., 2010). Nos homens, a relação entre a utilização dos esteroides anabolizantes e o sistema reprodutor, está ligado ao desencadeamento do câncer. Isso porque, os altos níveis de testosterona contribuem para o aparecimento do câncer de próstata (Nieman, 2011).

Outro efeito proveniente do consumo dos esteroides anabolizantes é a atrofia dos testículos (Nieman, 2011), que ocorre pelo consumo da testosterona sintética, onde o organismo suspende o comando de liberação de gonadotrofina pela hipófise e, consequentemente, as funções dos testículos, onde se fabrica a testosterona e os espermatozoides (Wilmore \& Costill, 2001).

Além disso, pode ocasionar ainda a esterilidade, tendo em vista a redução do número de espermatozoides (oligospermia), ausência de espermatozoides no sêmen (azoospermia) ou até mesmo desencadear uma impotência (Abrahin \& Sousa, 2013)

Já nas mulheres, os efeitos dos anabolizantes reduzem os níveis hormonais dos estrogênios e progesterona, alteração do ciclo menstrual, prolonga a amenorreia e causa a inibição da ovulação. Além disso, causa a hipertrofia do clitóris e pode provocar a atrofia do útero (Centro Regional de Informações de Medicamentos, 2000).

\section{Efeitos no sistema excretor}

Entre os órgãos que compõem o sistema urinário estão os rins. Os rins, tem como função o controle da concentração de substâncias no nosso sangue, são responsáveis ainda por garantir a excreção e a osmorregulação (controle das concentrações de sais nos tecidos e células). São eles que retiram os produtos do metabolismo celular da célula e as substâncias que se encontram em quantidade muito alta no organismo, garantindo, a homeostase dos líquidos corporais, que consiste na retirada desses produtos por meio da formação da urina (Robergs \& Roberts, 2002).

Mas, alguns compostos de esteroides parecem ter efeito adverso no funcionamento dos rins, isso porque o uso das substâncias sobrecarrega a funcionalidade dos rins, devido ao desequilíbrio causado nos eletrólitos e pressão sanguínea, apresentando efeitos colaterais maiores, quando o ciclo ou as doses administradas são elevados apresentando como sintomas como edema, dores, cólica renal, febre, aumento dos rins e inchaço na região costovertebral (Santos, 2007).

Outro problema recorrente é a glomerulonefrite, processo inflamatório nos rins que provoca perda de proteína e sangue pela urina. Essa condição pode evoluir para insuficiência renal crônica, quando os rins não conseguem mais desempenhar o seu papel de filtrar os líquidos e resíduos do organismo, causando o acúmulo dessas substancias e graves problemas a saúde, já que afeta a maioria dos sistemas e funções, incluindo a produção de glóbulos vermelhos, quantidade de vitamina D, controle da pressão arterial e saúde dos ossos (Robergs \& Roberts, 2002).

\section{Efeitos no sistema ósseo}

O osso, um tecido vivo, é uma forma rígida e altamente especializada de tecido conjuntivo, que compõe a maior parte do esqueleto e é o principal tecido de sustentação do corpo. Os ossos fornecem: Proteção para estruturas vitais; Suporte para o corpo e suas cavidades vitais; Base mecânica para o movimento; Armazenamento de sais; e, Suprimento contínuo de células sanguíneas novas (Moore, 2013).

Entre as doenças que mais acometem o sistema ósseo, está a osteoporose, que segundo a Organização Mundial da Saúde (OMS), é uma doença esquelética sistêmica progressiva, caracterizada por baixa massa óssea e deterioração microarquitetural do tecido ósseo, com consequente aumento da fragilidade óssea e suscetibilidade à fratura. 
A microarquitetura óssea tem a função de suportar os estresses mecânicos decorrentes no desenvolvimento das atividades diárias. Essa relação estrutura/função e a manutenção da homeostase mineral conduzem ao processo de formação e reabsorção do tecido ósseo, que perdura por toda a vida do indivíduo, denominado remodelação óssea (ROSSI, 2010).

Partindo dessa premissa, os esteroides anabolizantes podem causar danos à estrutura óssea, sendo observadas maior número de lesões nas articulações, cartilagens, meniscos e ligamentos (Abrahin \& Sousa, 2013).

$\mathrm{O}$ uso dos esteroides em adolescentes, pode desencadear distúrbios no crescimento e desenvolvimento ósseo. Isso porque, na puberdade os anabolizantes aceleram o fechamento das epífises (regiões dos ossos responsáveis pelo crescimento), reduzindo o período de crescimento, resultando em uma estatura menor do indivíduo (Bacurau, 2001). E, antecipando a fase final de crescimento (Calfee \& Fadale, 2006).

Além disso, a terapia estrogênica/terapia hormonal reduz a perda de tecido ósseo, aumenta a densidade tanto da coluna vertebral como no quadril, e reduz o risco de fraturas vertebrais e do quadril em mulheres na pós-menopausa. (Nieman, 2011).

\section{Ginecomastia}

A ginecomastia crescimento exagerado das mamas em homens, geralmente é causada pelo desequilíbrio hormonal, que pode ser de origem fisiológica (causada pelo próprio organismo), idiopática (causas desconhecidas) e patológicas (causada por alguma doença conhecida e efeito colateral de medicamentos e substâncias) (Mcardle \& Katch, 2000).

Isso ocorre quando durante o uso dos anabolizantes ocorre a diminuição da testosterona e aumento do estradiol em até sete vezes, quando comparados à média do hormônio encontrado nas mulheres, explicando assim o surgimento da ginecomastia (Mcardle \& Katch, 2000).

Como visto, o principal efeito colateral é a feminilização, afetando a estética e causando constrangimento. Mas, se submetido a tratamento precoce, a ginecomastia pode ser impedida (Santos, 2007).

\section{Outros efeitos}

Além dos já mencionados, outros efeitos adversos são recorrentes do uso de anabolizante. Entre eles, estão: dores de cabeça; tonturas; insônia; retenção de líquidos; sangramento no nariz; dores estomacais; estrias e alterações na pele ocasionando acne grave, com cicatrizes no rosto e corpo.

As dores articulares também são comuns, acrescido do risco aumentado de rompimento muscular, ruptura dos ligamentos, por arrancamento, e, rompimento de tendão, que se dá devido ao aumento excessivo dos músculos sem acompanhamento de crescimento necessário dos ligamentos.

Ressalta-se que a utilização dos esteroides anabolizantes por mulheres, implicam nas chances de masculinização do corpo. Isso porque há crescimento de pelos na face; na laringe, as cordas vocais são alteradas, deixando a voz mais grave ou rouca; além da alopecia (calvície de padrão masculino) que ocorre ao longo do uso das substâncias.

É importante dizer ainda que, esses hormônios não devem ser utilizados durante a gravidez, já que atravessam a barreira placentária e masculinizam o feto (Centro Regional de Informações de Medicamentos, 2000).

\section{Efeitos psicológicos e comportamentais}

Embora os efeitos fisiológicos serem os mais visíveis no uso dos anabolizantes, os efeitos psicológicos também tem uma presença bem marcante relacionado aos níveis de testosterona no corpo. Nesse sentido, podem causar oscilações na libido, alterações de humor e comportamental.

Os efeitos produzidos pelos esteroides anabolizantes são comparados aos produzidos por drogas psicoestimulantes, 
desencadeando um comportamento agressivo e hostil, antes do uso de esteroides anabolizantes. Isso se explica porque os anabolizantes atuam no sistema nervoso central, aumentando o metabolismo, o que implica um gasto exagerado de energia (Wilmore \& Costill, 2001).

Wilmore e Costill (2001) aponta a insatisfação com a imagem corporal um fator que mais predispõe à dependência. Mas, os efeitos variam de acordo com a utilização e doses de uso.

Segundo Santos (2003), uma patologia bem comum nesses casos, é denominada de vigorexia, também conhecido como transtorno disfórmico corporal, que se dá pelo distúrbio relacionado à percepção da imagem corporal, que pode até desenvolver ansiedade (Machado; Ribeiro, 2004). Os indivíduos com sintomas de vigorexia ao interromper o uso de esteroides passam a apresentar quadros de depressão, que passam a desenvolver dependência psicológica dos anabolizantes (Matos; Brandão, 2010). Wilmore e Costill (2001) afirma que, é no processo de retirada das substâncias que a síndrome de abstinência se apresenta. Entre os principais sintomas da abstinência, pode-se citar as alterações de humor, depressão, insônia, anorexia, diminuição da libido, fadiga, dor de cabeça, dores musculares e articulares (Diehl, Cordeiro \& Laranjeira, 2010).

Tendo em vista a gravidade dos efeitos no uso é importante ressaltar que em caso de manifestação dos sintomas, o indivíduo precisa procurar tratamento e apoio psicológico (Diehl, Cordeiro \& Laranjeira, 2010).

\section{Considerações Finais}

O presente trabalho trouxe os esteroides anabolizantes como objeto de estudo, analisando como as substâncias tem sido utilizadas de maneira errônea por praticantes de musculação, tendo em vista a busca do corpo perfeito idealizado pela mídia. Frente a isso, a pesquisa objetivou apresentar uma revisão de literatura quanto aos riscos da utilização de Esteroides Anabólicos no cenário da musculação, sem acompanhamento de um profissional qualificado.

Tal uso é corriqueiro devido os ganhos de massa corporal adquiridos pela administração dos esteroides, diminuindo assim o período estipulados para esses ganhos, uma vez que agem em sua maioria no aumento de força e consequentemente impacta em um maior volume de treino, que seria adquirido de forma mais gradual e lenta.

De um modo geral, os esteroides anabolizantes são utilizados para fins terapêuticos e sob acompanhamento médico, mas a grande preocupação está no uso em excesso, com finalidade diversa e sem prescrição profissional para uso. Isso porque, os efeitos adversos causam grandes problemas aos usuários, sobretudo podendo em sua maioria desencadear problemas crônicos e/ou irreversíveis.

Assim, é importante ressaltar que estudos mais voltados para essa temática são de grande valia tanto para equipe médica profissional, quanto para a população como um todo, uma vez que a informação permite a conscientização sobre o uso dos esteroides anabolizantes, frisando não somente seus benefícios, mas ressaltando ainda os efeitos colaterais que podem surgir quando utilizados de maneira demasiada.

Por fim, é importante mencionar que a base do tema em estudo pode ser explorada posteriormente, uma vez que durante a elaboração do trabalho percebeu-se a necessidade de uma abordagem mais detalhada acerca de alguns aspectos. Nesse sentido, fica como sugestão para trabalhos futuros, uma investigação sob forma de dados estatísticos da incidência dos principais efeitos causados em praticantes de musculação pelo uso dos anabolizantes a longo prazo, preenchendo assim lacunas existentes sobre essa informação do tema estudado.

\section{Referências}

Abrahin, O. S. C., \& Sousa, E. C. D. (2013). Esteroides anabolizantes androgênicos e seus efeitos colaterais: uma revisão crítico-científica. Revista da Educação Física/UEM, 24(4), 669-679. https://doi.org/10.4025/reveducfis.v24.4.17580

Bacurau, R. F. (2001). Hipertrofia: hiperplasia, fisiologia, nutrição e treinamento. São Paulo, Editora Phorte. 
Badillo, J. J. G., \& Ayestaran, E. G. (2004). Fundamentos do Treinamento de Força. São Paulo, Editora Manole.

Brinquinho, M., Sousa, A. S, Resende, J, \& Valente, J. (2017). Hepatotoxicidade associada ao uso de esteroides anabolizantes. Galicia Clin, 78(2), 79-81. http://dx.doi.org/10.22546/44/1060

Calfee, R., \& Fadale, P. (2006). Popular ergogenic drugs and supplements in young athletes. Pediatrics, 177(3), 577-589. https://doi.org/10.1542/peds.20051429

Carmo, E. C. do, Rosa, K. T., Koike, D. C., Fernandes, T., Silva Junior, N. D. da, Mattos, K. C., Wichi, R. B., Irigoyen , M. C. C., \& Oliveira , E. M. de. (2011). A associação de esteroide anabolizante ao treinamento aeróbio leva a alterações morfológicas cardíacas e perda de função ventricular em ratos. Revista Brasileira de Medicina do Esporte, 17(2), 137-141. https://doi.org/10.1590/s1517-86922011000200014

Carvalho, D. P, Fortunato, R. S., \& Rosenthal, Doris. (2007) Abuso de esteroides anabolizantes e seu impacto sobre a função tireóidea. Arquivos Brasileiros de Endocrinologia e Metabolgia, 51(9). https://doi.org/10.1590/S0004-27302007000900003

Centro Regional de Informações de Medicamentos. (2000). Esteroides Anabolizantes. http://www.farmacia.med.br/esteróides.htm.

Chagas, M. H, \& Lima, F. V. (2004). Variáveis estruturais: elementos primários para a sistematização do treinamento em musculação. In: Garcia E. S., Lemos, K.L.M. Temas Atuais em educação física e esportes. Silveira, 49-68.

Corsino, G. W. (2007). Variáveis da carga de treinamento na musculação: um estudo de revisão. Monografia (Graduação em Educação Física). UFMG.

Crozeta, C., \& Oliveira, G. K. (2009). Análise do perfil alimentar de mulheres com sobrepeso, praticantes de treinamento de força em academias de CuritibaPR. Revista Brasileira de Nutrição Esportiva, 3(17), 432-441. http://www.rbne.com.br/index.php/rbne/article/view/142

Diehl, A., Cordeiro, D.C., \& Laranjeira, R. (2010). Tratamento Farmacológicos para Dependência Química: Da evidência cientifica e a Prática Clínica. Artmed Editora S.A.

Estrela, C. (2018). Metodologia Científica: Ciência, Ensino, Pesquisa. Editora Artes Médicas.

Guimarães Neto, W. M. (2006). Musculação Além do Anabolismo. 2 ed. São Paulo, Editora Phorte.

Kersey, R. D., Elliot, D. L., Goldberg, L., Kanayama, G., Leone, J. E., Pavlovich, M., \& Pope, H. G. (2012). National athletic trainers' association position statement: Anabolic-androgenic steroids. Journal of athletic training, 47(5), 567-588. https://doi.org/10.4085/1062-6050-47.5.08

Lima, F. J., Correia, L. C. V, Almeida, B. B., Costa, L. A. S. \& Crisci, A. R. (2018). Efeitos do decanoato de nandrolona na função sexual de ratos wistar. Perspectivas Online: Biológicas \& Saúde, 8(27), 18-26. https://doi.org/10.25242/886882720181359

Machado, A. G., \& Ribeiro, P. C. P. (2004). Anabolizantes e Seus Risco. Adolescentes \& Saúde, 1(4), $20-22$.

Matos, A.P., \& Brandão, A. (2010). Perigo! Uma Bomba Chamada Anabolizante. Revista Pharmacia Brasileia, 57-60.

Nieman, D.C. (2011). Exercício e saúde: teste e prescrição de exercício. São Paulo, Editora Manole.

Oviedo, E. A. A. (2013). As Consequências do uso indevido dos esteroides anabolizantes androgênicos nas esferas civil, penal e administrativa: conhecer, prevenir, fiscalizar e punir. Brasília-DF.

Pedroso, R.C. Esteróides Anabólicos Androgênicos. In: Oga, S.; Camargo, M.M.A.; Batistuzzo, J.A.O. (2014). Fundamentos de Toxicologia. Atheneu, 557568 .

Robergs, R. A., Roberts, S. O. (2002). Princípios fundamentais de fisiologia do exercício para aptidão, desempenho e saúde. São Paulo, Phorte Editora.

Rossi, A. C., Freire. A.R., \& Menegati R. C. (2010). Osteoporose: considerações sobre terapêuticas atuais e metabolismo ósseo. International Journal of Dentistry dent, 9 (4). http://revodonto.bvsalud.org/scielo.php?script=sci_arttext\&pid=S1806-146X2010000400008

Schoenfeld, B. J., Peterson, M. D., Ogborn, D., Contreras, B., Sonmez, G. T. (2015). Effects of Low- vs. High-Load Resistance Training on Muscle Strength and Hypertrophy in Well-Trained Men. Journal Of Strength And Conditioning Research, 29(10), 2954-2963. https://doi.org/10.1519/jsc.0000000000000958

Silva, P. R. P, Machado Junior L. C, Figueiredo V. C, Cioffi A. P, Prestes M. C, \& Czepielewski M. A. (2007). Prevalência do uso de agentes anabólicos, em praticantes de musculação de Porto Alegre. Arq. Bras. Endocrinol. Metab., 51(1), 104-10. https://doi.org/10.1590/S0004-27302007000100017

Silva, P. R. P. D., Danielski, R., \& Czepielewski, M. A. (2002). Esteróides anabolizantes no esporte. Revista Brasileira de Medicina do Esporte, 8(6), 235243. https://doi.org/10.1590/S1517-86922002000600005

Alves Sobrinho, C., Rossi Junior, W. C., Corsini, W., Soares, E. A., \& Esteves, A. (2020). Doses suprafisiológicas de esteroides anabolizantes e os efeitos no coração de ratos jovens sedentários: estudo morfométrico. Research, Society and Development, 9(11), e72091110079. https://doi.org/10.33448/rsdv9i11.10079

Sousa, R. V. (2002). Efeitos do uso de esteroides anabolizantes. Monografia (graduação em Ciências Biológicas). Faculdade de Ciências da Saúde. Uniceub.

Venâncio, D. P., Nobrega, A. C. L. da, Tufik, S., \& Mello, M. T. (2010). Avaliação descritiva sobre o uso de esteroides anabolizantes e seu efeito sobre as variáveis bioquímicas e neuroendócrinas em indivíduos que praticam exercício resistido. Revista Brasileira de Medicina do Esporte, 16(3), 191-195. https://doi.org/10.1590/S1517-86922010000300007

Tortora, G. J., \& Derrickson, B. (2017). Corpo Humano: fundamentos de anatomia e fisiologia. 10ª ed. São Paulo. Editora Artmed.

Wilmore, J. H., \& Costill, D. L. (2001). Fisiologia do esporte e do exercício. 2.ed. São Paulo, Editora Manole. 